International Journal of Pediatric Otorhinolaryngology

60 (2001) 113-118

\title{
Auditory abnormalities associated with unilateral renal agenesis
}

\author{
Hung-Meng Huang a, Re-Ming Yeh ${ }^{\mathrm{b}}$, Ching-Ting Tan ${ }^{\mathrm{c}}$, Min-Cheng Chao ${ }^{\mathrm{a}}$, \\ Kai-Nan Lin ${ }^{\mathrm{c}, *}$ \\ a Department of Otorhinolaryngology, Taipei Municipal Women and Children Hospital, 12, Fu Cho St, Taipei, Taiwan, ROC \\ b Department of Otorhinolaryngology, Chang Gung Memorial Hospital, 5, Fu-Shin St, Gau-Shan, Tau-Yuan 333, Taiwan, ROC \\ c Department of Otolaryngology, National Taiwan University Hospital, 1, Cheng-Te St, Taipei, Taiwan, ROC
}

Received 24 January 2001; received in revised form 23 April 2001; accepted 24 April 2001

\begin{abstract}
Objective: To evaluate the relationship between unilateral renal agenesis and auditory abnormality, and to determine the clinical spectrum of hearing impairment in such patients. Methods: Seventy-five children with unilateral renal agenesis underwent auditory examinations. The subjects comprised 35 males and 40 females. Fourteen females had mullerian abnormalities. Another 75 schoolchildren with the same gender profile were selected for audiometric testing as a control group. Children with sonographically evident urogenital system abnormalities were excluded from the control group. Results: The prevalence of auditory abnormalities in children with unilateral renal agenesis $(4 / 75)$ $(5.3 \%)$ was higher than in the control group $(0 \%)$. The prevalence in children with urogenital anomalies was significantly higher in patients with renal agenesis than in the normal population (28.5\%). Audiometric results showed that four of the 75 children manifested ipsilateral sensorineural hearing impairment, particularly in the high-frequency range. All were females with coexisting genital abnormalities. Two were diagnosed with mild sensorineural hearing impairment while the other two had moderate hearing loss. Conclusions: The results of our study suggest that neurosensory hearing loss was found to be associated with renal agenesis. Further audiometric follow-up of children with renal agenesis seems worthwhile. (C) 2001 Elsevier Science Ireland Ltd. All rights reserved.
\end{abstract}

Keywords: Renal agenesis; Unilateral; Auditory abnormalities

\section{Introduction}

To establish the prevalence of various renal abnormalities in school children, Hsieh et al. [1] conducted an epidemiological study with real-time

\footnotetext{
* Corresponding author. Tel.: 886-2-23916471x295; fax: 8862-23519548.

E-mail address: orl_fu@health.gov.tw (K.-N. Lin).
}

ultrasound screening. In total, 103 children with unilateral renal agenesis were identified, of which 75 were referred to our department for screening aimed at the detection of audiological abnormalities. Given that the auditory system and urogenital system develop in the same embryonic period and that some specific syndromes [2-5] have both auditory and urogenital anomalies, this study attempted to determine the spectrum of auditory 
defects that exists in association with unilateral renal agenesis.

\section{Materials and methods}

Seventy-five children with unilateral renal agenesis confirmed by renal sonography and intravenous pyelography underwent audiometric testing to assess their hearing function. The group comprised 35 males and 40 females with ages ranging between 6 and 16 years. Thirty-seven had left sided renal agenesis and 38 suffered from right sided renal agenesis. Amongst the female subjects, 14 had a double uterus identified on hysterosalpingography (HSG) (Table 1). Hydrocolpus and hematocolpus were found in two of these 14 children respectively. Vaginal septectomies were performed to correct these structural abnormalities. Seventy-five children free of sonographically identifiable urogenital abnormalities were selected with the same gender profile as a control group.

A questionnaire enquiring after family history, maternal systemic diseases, maternal age, viral infection and any drug history during pregnancy was performed for all 75 children. Otoscopic examination was performed before audiometric testing to identify any external ear abnormalities.
Audiometric testing was performed in a doublewalled, sound-treated booth with a diagnostic audiometer. Each child was evaluated as follows: (1) pure tone air and bone conduction thresholds for the octave frequencies $250-8000 \mathrm{~Hz}$; (2) tympanometry; (3) acoustic contralateral and ipsilateral reflex measurements $(500,1000,2000,4000 \mathrm{~Hz})$, and finally; (4) reflex decay measurements at 500 and $1000 \mathrm{~Hz}$. The normal threshold intensity for each frequency was a hearing level of $<25 \mathrm{~dB}$. Threshold ranges for mild, moderate, severe and profound hearing loss were defined as 25-40, $40-55,55-70$ and $>70 \mathrm{~dB}$, respectively. The results were analysed with chi-square test.

\section{Results}

No obvious external ear abnormalities were found in either of the two groups of children. The prevalence of hearing impairment in the children with unilateral renal agenesis was 5.3\% (4/75). It was higher than the rate of the control group $(0 \%)$ although the chi-square test didn't reveal significantly. The prevalence of hearing impairment in the children with urogenital abnormalities was even higher than normal population $(28.5 \%)(4 /$

Table 1

Multiple abnormalities in patients with unilateral renal agenesis

\begin{tabular}{llllll}
\hline Patient No. & Sex & Age $(\mathrm{y} / \mathrm{o})$ & Genital anomaly & $\begin{array}{l}\text { Side of renal } \\
\text { agenesis }\end{array}$ & Auditory anomaly \\
\hline 1 & & & & $\mathrm{~L}$ & $(-)$ \\
2 & $\mathrm{~F}$ & 12 & Double uterus & $\mathrm{R}$ & $(-)$ \\
3 & $\mathrm{~F}$ & 15 & Double uterus & $\mathrm{R}$ & $(-)$ \\
4 & $\mathrm{~F}$ & 8 & Double uterus & $\mathrm{R}$ & $(-)$ \\
5 & $\mathrm{~F}$ & 12 & Double uterus & $\mathrm{R}$ & $(-)$ \\
6 & $\mathrm{~F}$ & 12 & Double uterus & $\mathrm{L}$ & $(-)$ \\
7 & $\mathrm{~F}$ & 16 & Double uterus & $\mathrm{R}$ & $(-)$ \\
8 & $\mathrm{~F}$ & 14 & Double uterus & $\mathrm{L}$ & $(-)$ \\
9 & $\mathrm{~F}$ & 16 & Double uterus & $\mathrm{R}$ & $(-)$ \\
10 & $\mathrm{~F}$ & 10 & R Double uterus & $\mathrm{L}$ & $(-)$ \\
11 & $\mathrm{~F}$ & 14 & Double uterus & $\mathrm{R}$ & $\mathrm{M}_{1}{ }^{\mathrm{b}} \mathrm{SNHL}^{\mathrm{a}}$ \\
12 & $\mathrm{~F}$ & 6 & Double uterus & $\mathrm{R}$ & $\mathrm{M}_{1}^{\mathrm{b}} \mathrm{SNHL}^{\mathrm{a}}$ \\
13 & $\mathrm{~F}$ & 16 & Double uterus & $\mathrm{R}$ & $\mathrm{M}_{2}^{\mathrm{c}} \mathrm{SNHL}^{\mathrm{a}}$ \\
14 & $\mathrm{~F}$ & 13 & Double uterus c hematocolpos & $\mathrm{M}_{2}^{\mathrm{c}} \mathrm{SNHL}^{\mathrm{a}}$
\end{tabular}

\footnotetext{
${ }^{a}$ SNHL: Sensorineural Hearing Loss.

${ }^{\mathrm{b}} \mathrm{M}_{1}$ : mild.

${ }^{\mathrm{c}} \mathrm{M}_{2}$ : moderate.
} 
Table 2

Threshold responses in patients c urogenital abnormalities

\begin{tabular}{|c|c|c|c|c|c|c|c|c|}
\hline \multirow[t]{2}{*}{ Patient } & \multicolumn{8}{|c|}{ Frequency $(\mathrm{Hz})$ (right ear/left ear) } \\
\hline & 250 & 500 & 1000 & 2000 & 3000 & 4000 & 6000 & 8000 \\
\hline 2 & $20 / 25$ & $25 / 20$ & $25 / 20$ & $30 / 25$ & $30 / 25$ & $35 / 20$ & $40 / 15$ & $40 / 25$ \\
\hline 3 & $15 / 10$ & $5 / 15$ & $15 / 15$ & $40 / 20$ & $35 / 25$ & $40 / 20$ & $55 / 15$ & $50 / 15$ \\
\hline
\end{tabular}

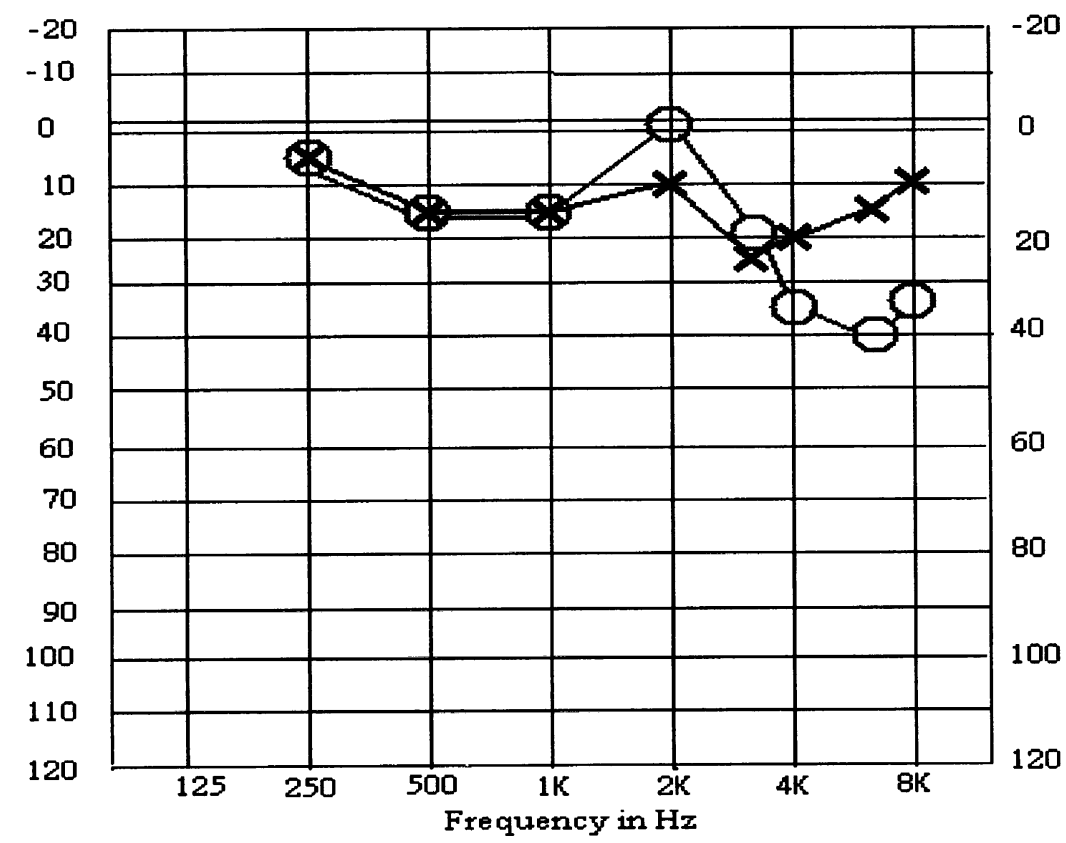

Fig. 1. The audiogram for case 1 in Table 2 showing mild high pitched sensorineural hearing impairment.

14) (Table 2). The chi-square test showed significantly $(P<0.05)$. The four children were all female and two of them were diagnosed with ipsilateral mild sensorineural impairment (Figs. 1 and 2). The other two were diagnosed with ipsilateral moderate sensorineural impairment (Figs. 3 and 4). No significant findings were noted with other audiometric testing that included tympanometry, contralateral and ipsilateral acoustic reflex measurements and reflex decay measurement.

Other than renal agenesis, no risk factors in the areas of family history, maternal age, history of infection, drug exposure, etc were present in patients with hearing loss.

\section{Discussion}

In 1946, Potter [6] first examined the relationship between external ear abnormalities and renal agenesis. Taylor [7] indicated that six of his 10 patients with gross external ear deformity had a congenital abnormality of the kidney or upper urinary tract. Notwithstanding this, no external ear abnormalities were found in the seventy-five 


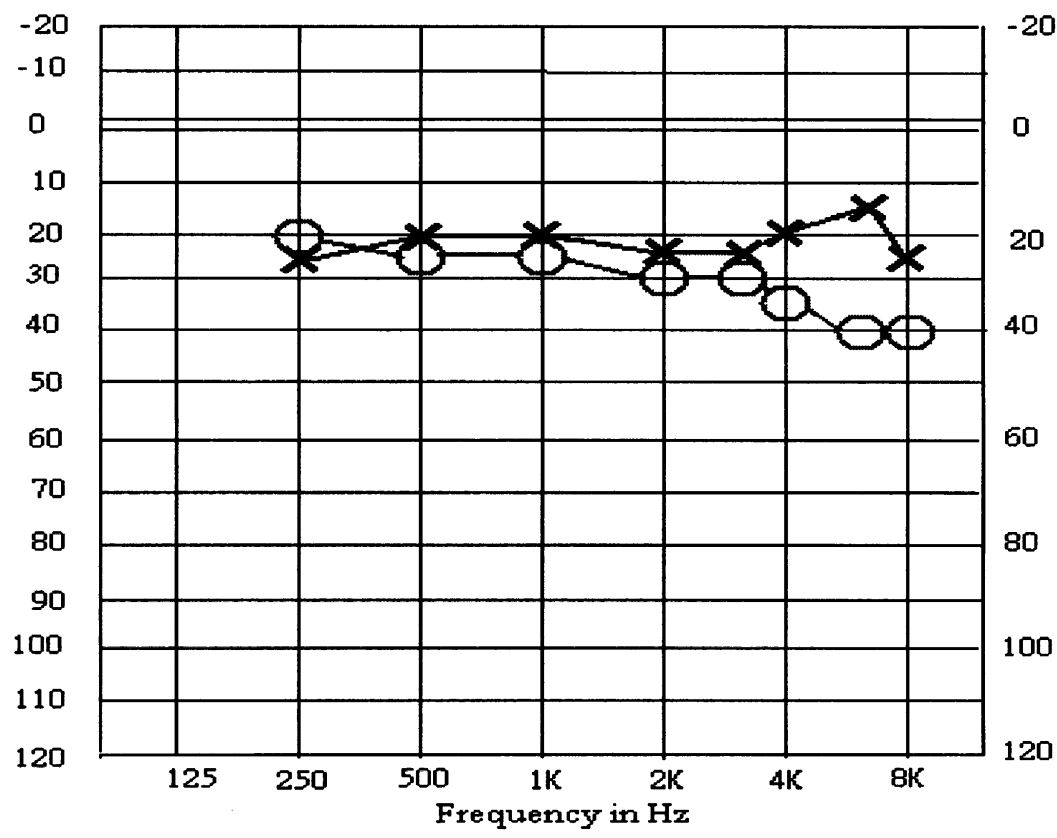

Fig. 2. The audiogram for case 2 in Table 2 showing mild high pitched sensorineural hearing impairment.

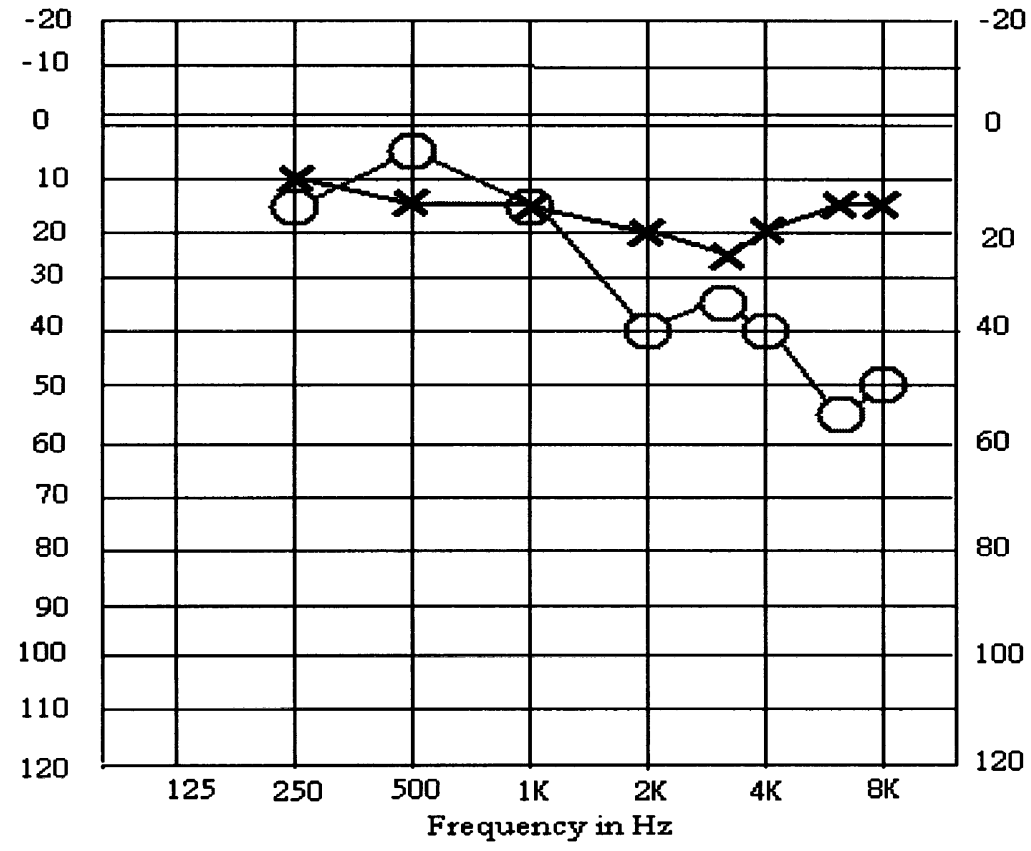

Fig. 3. The audiogram for case 3 in Table 2 showing moderate high pitched sensorineural hearing impairment. 


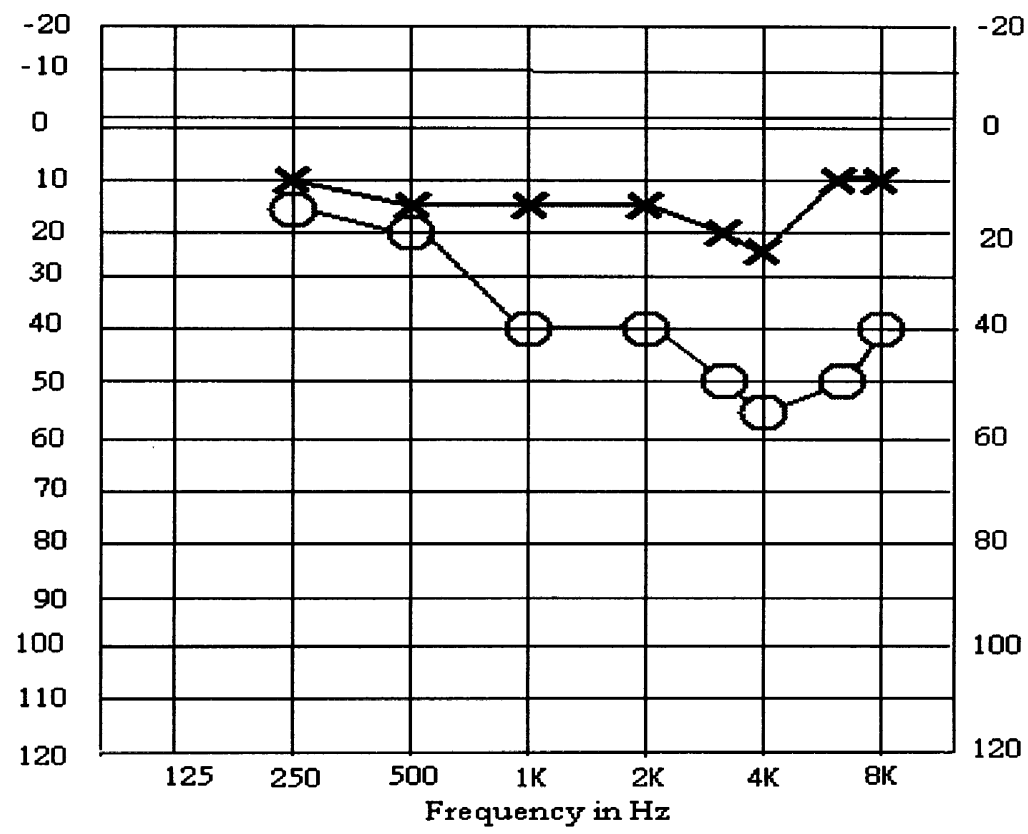

Fig. 4. The audiogram of case 3 in Table 2 showing moderate high pitched sensorineural hearing impairment

children with unilateral renal agenesis investigated in this study.

The prevalance of auditory abnormalities in children with unilateral renal agenesis was $5.3 \%$, similar to Bergstrom's result. [8]. It reveals that congenital hearing loss may be more common in children with renal disorders. Embryologically, the urogenital and auditory systems begin development at 4-6 weeks, with completion at around 16-18 weeks [9]. Teratogenic influences during this period may result in a combination of abnormalities of the urogenital and auditory systems. Earlier teratogenic insults may result in severe and complete system defects. A double uterus is typically due to an early insult. Two of the 14 female children with unilateral renal agenesis and a double uterus manifested mild ipsilateral sensorineural hearing impairment, ranging from 3000-8000 $\mathrm{Hz}$. The remaining two females exhibited moderate ipsilateral sensorineural hearing impairment. The degree of hearing loss in the four children seemed to parallel the severity of their mullerian and renal system anomalies. None of the 35 male children had auditory or genital abnormalities. Gender may affect the response to teratogenic insults in this period and further investigation seems worthwhile.

Letterie [10] indicated that five of 15 females investigated with genital abnormalities also suffered from mild to moderate sensorineural hearing impairment. Four of the 15 patients had coexisting unilateral renal agenesis. The results of this study would suggest that the urinary system is the most vulnerable of the three systems, while the auditory system is the system most resistant to teratogenic insults in this period. Racial differences may explain the differences seen between the two studies.

\section{Conclusion}

The results of the study suggest that the severity auditory system defects parallel those of the urogenital system. Auditory defects may not always be clinically evident and identification may require audiometric testing. Dynamic audiometric tests are appropriate for patients with anomalies of the urogenital system, regardless of the severity of the anomaly. Periodic audiometric tests are 
also appropriate for the identification of any deterioration in hearing if audiometric defects are detected in conjunction with anomalies of the urogenital system.

\section{Acknowledgements}

The role played by Dr CP Hsieh in referring patients to our department for audiological evaluation is appreciated.

\section{References}

[1] C.P. Shieh, M.B. Liu, C.S. Huang, K.H. Yang, W.Y. Chen, C.Y. Lin, Renal abnormalities in schoolchildren, Pediatrics 84 (1989) 1068-1090.

[2] J.S.D. Winter, G. Roan, W.J. Mellmen, et al., A familial syndrome of renal, genital and middle ear abnormalities, J. Pediatr. 72 (1968) 88-93.
[3] G. Turner, A second family with renal, vaginal and middle ear abnormalities, J. Pediatr. 76 (1970) 641.

[4] L.A. King, L. Sanchez-Ramos, O.E. Talledo, et al., Syndrome of genital, renal and middle ear abnormalities. A third family and report of a pregnancy, Obetet. Gyneco. 69 (1987) 491-493.

[5] B.H. Pennie, H.A. Marres, Shoulder abnormalities in association with branchio-oto-renal dysplasia in patient who also has familiar joint laxity, Int. J. Pediatr. Otorhinolaryngol. 23 (3) (1992) 269-273.

[6] E.L. Potter, Bilateral renal agenesis, J. Pediatr. 29 (1946) 68-76.

[7] W.C. Taylor, Deformity of ears and kidneys, Canad. Med. Ass. J. 93 (1965) 107-110.

[8] L. Bergstrom, P. Thompson, Hearing loss in pedriatric renal patients, Int. J. Pediatr. Otorhinolaryngol. 5 (3) (1983) 227-234.

[9] K.L. Moore, The Developing Human: Clinically Oriented Embryology, WB Saunders, Philadelphia, 1991.

[10] M.G.S. Letterie, M.N. Vauss, Mullerian tract abnormalities and associated auditory defects, The Journal of Reproductive Medicine 36 (1991) 765-768. 